\title{
Visibilidad internacional del CACl: actividad científica
}

\author{
CACl international visibility: scientific activity
}

Revista Argentina de Cardioangiologia Intervencionista 2019;10(4):0190-0191. https://doi.org/10.30567/RACI/201904/0190-0191

Estimados colegas y amigos, es para mí una gran satisfacción ponerme una vez más en contacto con ustedes a través de este nuevo número de nuestra revista.

En números previos me he referido, en este espacio, a algunos de los diferentes proyectos e iniciativas, sobre los cuales hemos trabajado los miembros de Comisión Directiva y de diferentes áreas y comisiones del Colegio, durante los dos últimos años. Por mencionar algunos:

1. Remodelación del 5 to piso de nuestra sede e inauguración del Centro de Simulación del CACI.

2. Concreción y publicación de los tomos II y III del E-Book de Hemodinamia y Cardioangiología Intervencionista.

3. Creación de la Agencia de Innovación del CACI.

4. Organización de la primera edición de Innovations in Cardiovascular Interventions (ICI - CACI) en Latinoamérica, a llevarse cabo el 4 de agosto de 2020.

5. La 4ta Jornada CACI para Financiadores y Auditores médicos; Primera Jornada CACI para la Industria; Primera Jornada CACI - ANMAT, la cual fortaleció aún más nuestras relaciones con la entidad regulatoria nacional.

En la carta que tuve oportunidad de escribir en el primer número del año 2018 de la Revista Argentina de Cardioangiología Intervencionista (RACI), hice referencia a algunos de los objetivos que la Comisión Directiva 2018 - 2019, que tuve el honor de presidir, se planteó para la gestión.

Entre estos objetivos, me referí a la necesidad de afianzar las relaciones de nuestro Colegio con las sociedades científicas nacionales e internacionales, manteniendo y afianzando la participación del CACI, con sesiones propias o conjuntas con otras sociedades científicas, en los congresos más prestigiosos de nuestra especialidad.

Tal como enuncia nuestro Estatuto en su artículo 2, dentro de los múltiples objetivos y fines de nuestro Colegio se contempla la participación en congresos nacionales e internacionales, en representación del sector.

El trabajo incansable, la capacidad de gestión y la tenacidad de muchos socios del Colegio y de Comisiones Directivas previas comenzó a dar sus frutos, con la primera sesión CACI@TCT en 2008; a partir de 2013 se agregaron las sesiones CACI@EuroPCR; desde el 2015 sumamos sesiones CACI@C3 (Complex Cardiovascular Catheter Therapeutics) en Orlando, EE.UU. Extendiéndonos más allá de Estados Unidos y Europa, desde 2017 estamos presentes en China con la sesión CACI@CIT (China Interventional Therapeutics), y a partir de 2018 en India, con la sesión CACI@India Live, de tal forma que, en la actualidad, CACI está presente con participación científica, a lo largo y a lo ancho del planisferio, siendo la única sociedad científica de cardiología intervencionista de Latinoamérica con esta participación y visibilidad internacional.

Esta intensa actividad científica internacional ha permitido, a su vez, tan solo durante los años 2018-2019, la participación de 79 socios del Colegio en estos congresos, cumpliendo roles de disertantes, coordinadores, presentadores de casos o panelistas, desempeñándose como embajadores de la Cardioangiología Intervencionista Argentina en esta multiplicidad de eventos.

No puedo dejar de mencionar y reconocer el trabajo y el mérito de los miembros de la Oficina de Congresos CACI, para que el programa de cada una de estas sesiones sea original y atractivo para los asistentes.

No satisfechos con haber logrado esta visibilidad internacional del CACI, tengo el orgullo de compartir con ustedes que, a través de la acción de la Comisión Directiva, hemos logrado gestionar durante estos dos últimos años becas para congresos internacionales para los socios del Colegio, a saber, 7 becas completas para el Congreso TCT, 1 beca completa para el Congreso EuroPCR 2019 y 4 becas que incluyeron inscripción y alojamiento para el Congreso C3 2019 en Orlando.

Como se anunció en la convocatoria general, fueron requisitos excluyentes para aplicar a una de estas becas ser socio CACI Activo o Adherente, con las obligaciones para con el Colegio al día, dándose además prioridad a los postulantes con casos o abstracts aceptados para ser presentados en dicho congreso que no contaran con apoyo para concurrir a presentar sus trabajos, en reconocimiento al esfuerzo que implica la preparación y aceptación de un abstract o caso desafiante para ser presentado en un congreso internacional.

Este crecimiento en la participación del CACI en los congresos internacionales de la especialidad se produjo sin descuidar nuestra presencia en los congresos nacionales de Cardiología. Por décimo año consecutivo, se desarrolló el X Simposio CACI@FAC-VII Sesión conjunta CACI-GACI en el marco del XXXVI Congreso Nacional de Cardiología, que tuvo lugar en la ciudad de Rosario el 31 de mayo de 2019. En esta oportunidad, contamos con la participación de varios invitados internacionales en nuestra relación con el grupo GACI francés (Dres. Hakim Benamer, Gerard Souteyrand, Gregoire Range y Eduardo Aptecar), y tuvimos un gran número de asistentes. 
El 18 de octubre de este año participamos del $45^{\circ}$ Congreso Argentino de Cardiología, en el Predio Ferial "La Rural” de la ciudad de Buenos Aires, donde organizamos el XI Simposio TCT@CACI@SAC con la participación de los Dres. Fayaz Shawl y Hemal Gadda, entre otros, y una videoconferencia desde EE.UU. del Dr. Juan Granada (CRF). Esta actividad se llevó a cabo en el Salón García Marquez, con capacidad para 300 personas, el cual contó con una nutrida concurrencia durante todo el Simposio. Por primera vez en este congreso utilizamos un lector óptico que nos permitió conocer el perfil de los asistentes a nuestro simposio, resultando de gran interés el hallazgo que, de 480 asistentes al Simposio, 64\% eran clínicos o cardiólogos clínicos, lo cual enfatiza seguramente la importancia de la presencia de nuestro Colegio en estos eventos.

No quiero dejar pasar la oportunidad de mencionarles, que en virtud del trabajo del Área de Investigación del Colegio, en septiembre de 2019 comenzó la inclusión de pacientes en el Registro Argentino de Angioplastia Coronaria - RAdAC 2, coordinado en forma conjunta por el CACI, el Consejo de Hemodinamia de la SAC y el Comité de Hemodinamia de la FAC.

El objetivo fundamental de RAdAC 2 es conocer cómo estamos realizando nuestras intervenciones coronarias en la actualidad, ya que es notable en los últimos años la evolución de las indicaciones y recursos disponibles.

En septiembre de 2019 comenzó la inclusión de pacientes en el registro. Se incorporaron para participar 53 centros médicos de 17 provincias, habiéndose incluido 535 procedimientos hasta el 15 de noviembre de 2019.

Los invito a todos a que, como miembros del Colegio, nos comprometamos a tener una participación activa en este registro, el cual de esa manera representará el abanico completo de la realidad de la práctica de las intervenciones coronarias en nuestro país.

Por último, y en nombre de la Comisión Directiva 2018-2019 que tuve el honor de presidir, quiero agradecerles a cada uno de ustedes la confianza y el apoyo a nuestra gestión durante estos dos años. No duden de que pusimos todo nuestro empeño y esfuerzo para dejar nuestra pequeña huella en la historia de nuestro querido Colegio. 